\title{
Eficiencia energética dentro del sistema municipal de parques temáticos de Tijuana
}

\section{Energy efficiency within the municipal system of Tijuana theme parks}

\author{
TEÓN-VEGA, Argelia†*, VARGAS-GURROLA, Luis Eduardo, MONTAÑO-SOTO, Myriam Tatiana \\ y BALDERAS-LÓPEZ, Silvia María
}

Universidad Tecnológica de Tijuana, Dirección de Tecnología Ambiental y Energías Renovables, México.

ID $1^{\text {er }}$ Autor: Argelia, Teón-Vega / ORC ID: 0000-0003-2274-6367, CVU CONACYT ID: 412627

ID $1^{\mathrm{er}}$ Coautor: Luis Eduardo, Vargas-Gurrola / ORC ID: 0000-0001-8456-2948, CVU CONACYT ID: 179573

ID $2^{\text {do }}$ Coautor: Myriam Tatiana, Montaño-Soto / ORC ID: 0000-0003-0741-9173, CVU CONACYT ID: 324049

ID $3^{\text {er }}$ Coautor: Silvia María, Balderas-López / ORC ID: 0000-0003-0434-5893, CVU CONACYT ID: 273260

DOI: $10.35429 / J U S D .2020 .18 .6 .28 .35$

Recibido: 20 de Enero, 2020; Aceptado 30 de Junio, 2020

\section{Resumen}

El parque Morelos forma parte del Sistema Municipal de Parques Temáticos de Tijuana (SIMPATT). Debido a las múltiples actividades que se realizan para la comunidad, así como la falta de mantenimiento a la instalación eléctrica, entre otros factores, el coste por consumo de electricidad anda alrededor de \$1, 022,107.00 M.N. anuales. Objetivo: el propósito del presente proyecto es realizar un estudio de eficiencia energética para la obtención de indicadores de desempeño energético (KPI) con los cuales se pueda proponer mejoras para el uso racional y eficiente de la energía. Metodología: el proyecto se trabajó con la colaboración de alumnos en estadía de la ingeniería en Energías Renovables. El estudio comprendió las siguientes etapas: 1) diagnóstico energético para la determinación del estado actual en el consumo de energía eléctrica; 2) análisis de la información obtenida para la detección de áreas de mejora; y 3) propuestas para un uso eficiente de la energía eléctrica. Contribución: con el desarrollo de este estudio, además de proporcionar un beneficio económico viable a la administración del parque, se contribuye a la integración y aplicación de conocimiento de futuros ingenieros en un ámbito real.

\begin{abstract}
Morelos Park is part of the Municipal System of Tijuana Theme Parks (SIMPATT). Due to the multiple activities carried out for the community, as well as the lack of maintenance to the electrical installation, among other factors, the cost for electricity consumption is around $\$$ $1,022,107.00$ M.N. yearly. Objective: the purpose of this project is to carry out an energy efficiency study to obtain energy performance indicators (KPI) with which improvements for the rational and efficient use of energy can be proposed. Methodology: the project was worked with the collaboration of students in renewable energy engineering program internship. The study includes the following stages: 1) gathering energy data to determine the current state of electrical energy consumption; 2) analysis of the information obtained inorder to detect improvement areas; and 3) proposals for an efficient use of electrical energy. Contribution: with the development of this study, in addition to providing a viable economic benefit to the park administration, it contributes to the integration and application of tools and knowledge of future engineers in real environment situations.
\end{abstract} Revista del Desarrollo Urbano y Sustentable. 2020. 6-18:28-35.

\footnotetext{
*Correspondencia al autor (Correo electrónico: argelia.teon@uttijuana.edu.mx)

$\dagger$ Investigador contribuyendo como primer autor.
} 


\section{Introducción}

En la actualidad, el consumo energético es uno de los mayores contribuidores de emisiones contaminantes a la atmósfera por el uso intensivo de energéticos fósiles para la generación de energía eléctrica. En México, las emisiones totales del sector energético reportadas se contabilizan en $522 \mathrm{MtCO} 2 \mathrm{e}$ (INEGyCEI 2017). De acuerdo con la Convención Marco de las Naciones Unidas sobre el Cambio Climático (CMNUCC) en el Acuerdo de París de 2015, ratificado en 2019, la Contribución Nacional Determinada (NDC, por sus siglas en inglés) de México debe de disminuir en un $25 \%$ sus emisiones de GEI para el año 2030.

Con la finalidad de contribuir a la disminución de las emisiones de GEI, se han propuesto diferentes mecanismos para la mitigación de GEI, tales como: incorporación de energías renovables, uso eficiente de la energía, protección de bosques, buenas prácticas agrícolas, transporte limpio, gestión de residuos y mejora de procesos industriales. La utilización eficiente de energéticos es primordial tanto en los procesos de generación de energía, como en los procesos productivos en industria. En este sentido, el Programa de Desarrollo del Sector Eléctrico Nacional (PRODESEN 2019-2033) de la Secretaría de Energía, a través de la Comisión Nacional del Uso Eficiente de la Energía, (CONUEE) establece estrategias y hojas de ruta de eficiencia energética para la reducción de emisiones.

En materia de eficiencia energética, los usos eficientes dentro de edificios y servicios públicos representan una gran área de oportunidad ya que son usuarios que presentan comportamientos lineales y predecibles [ref art]. Además de que el pago del servicio eléctrico de estos proviene de recurso público y debe de ser parte de políticas públicas que el uso eficiente se reglamente en estos. Los usuarios como lo son espacios públicos, ya sea parque o plazas no son la excepción.
La ciudad de Tijuana, Baja California, alberga dos grandes parques. Parque de la Amistad, en la delegación de OTAY y el Parque Morelos ubicado en la delegación de La Mesa. Ambos cuentan con una administración pública $\mathrm{y}$ en ambos casos se distingue un crecimiento en actividades para cubrir las demandas de los visitantes. La mayor parte de las actividades derivan en servicios cuyo consumo energético no es regulado por alguna entidad dentro de la administración del parque. Un diagnóstico energético y un balance de energía son requeridos. Las herramientas para emplear en la elaboración del presente trabajo son el diagnóstico energético tipo DEN I, lo cual es recomendado dentro de las guías de la CONUEE como las primeras prácticas para abordar el tema de eficiencia energética. Se llevará a cabo un monitoreo de calidad de energía para elaborar Indicadores clave de desempeño con base a eficiencia energética y finalmente se elaborarán propuestas de mejora a partir de los datos obtenidos.

El desarrollo de estas herramientas dentro de un estudio de eficiencia energética permite caracterizar consumos energéticos hasta un nivel de usuario final por área $\mathrm{o}$ departamento. Lo cual contribuye a generar una herramienta operativa cuyos resultados pueden ser medidos para proyectos de mejora y que ofrece indicadores para seguimiento de su desempeño.

Esto enfocado a abordar la problemática de una planeación insuficiente para cubrir los servicios eléctricos dentro de un Parque Temático Municipal el cual presenta áreas de oportunidad para ahorro de energía. Este estudio se llevó a cabo con la finalidad de que se adopten medidas e indicadores de desempeño energético dentro de las instalaciones que permitan trazar metas y objetivos para sus diversas actividades. De esta manera será un parque que cuente con buenas prácticas energéticas. En las subsecuentes secciones se abordará el contexto del sujeto de estudio, en este caso el parque Morelos del SIMPATT, posteriormente se analizará la metodología propuesta donde se expone el diagnóstico energético, análisis de información y propuestas de mejora. Los principales hallazgos se discuten en los resultados y posteriormente las conclusiones aportan los principales hitos en el estudio. 


\section{Contexto del estudio}

El parque José María Morelos y Pavón, comúnmente conocido como parque Morelos, forma parte del Sistema Municipal de Parques Temáticos de Tijuana (SIMPATT), el cual es un organismo descentralizado que tiene como objetivo mantener, crear y promover espacios de áreas verdes conformadas por recursos naturales e infraestructura de servicios con los fines de mejorar el ambiente, embellecer el paisaje urbano y ofrecer a la población espacios de descanso, recreo y entretenimiento.

En el contexto eléctrico del parque Morelos, las instalaciones eléctricas del mismo se encuentran en mal estado, debido a que ha contado a través de los años con crecimiento en sus actividades y servicios fuera de su planeación inicial, lo que ha derivado en instalaciones eléctricas que no cumplen con normativas, así como por la falta de mantenimiento preventivo. Áreas de oportunidad como documentación de planos y diagramas, señalización son otras, así como concesionarios o locatarios que se encuentran conectados a la red eléctrica interna de manera insegura lo cual presenta ambos un riesgo y un foco de ineficiencia en el uso de energía, sin menoscabar que el pago por la energía para estos servicios no es adecuado a la tarifa en la que se encuentra el Parque de manera general.

\section{Metodología a desarrollar}

La metodología implementada en el presente trabajo incluye tres etapas, cada una de ellas con sus respectivas actividades, las cuales se muestran en el diagrama de la figura 1 :

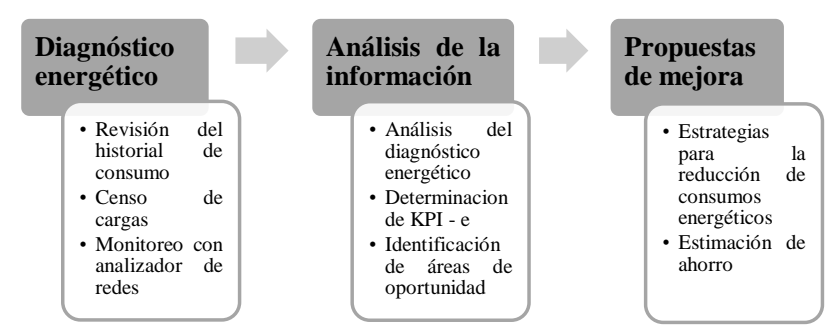

Figura 1 Diagrama de proceso de las etapas del proyecto Fuente: Elaboración propia

\section{Diagnóstico energético}

\section{Revisión del historial de consumo energético}

Se inició con una revisión del historial de consumo energético, partiendo del recibo de facturación eléctrica, esto con la finalidad de verificar el comportamiento de consumo de al menos un año. En esta etapa es posible identificar en cuáles meses existe un mayor consumo energético.

\section{Censo de cargas}

Se realizó un censo de cargas eléctricas para determinar los perfiles de consumo de las áreas del parque, incluyendo entrevistas con el personal operativo con la finalidad de conocer el uso de este y de esta manera dividir en tanto departamentos o áreas, y los usos significativos de energía (USE). Para dicha actividad, se tomó como base la "Guía para elaborar un diagnóstico energético en inmuebles" de la CONUEE (CONUEE, 2019), concentrando los datos en un formato como el que se muestra en la tabla 1.

\begin{tabular}{|c|c|c|c|}
\hline Concepto & $\begin{array}{c}\text { Consumo } \\
\text { mensual } \\
(\mathrm{kWh} / \mathrm{mes})\end{array}$ & $\begin{array}{c}\text { Consumo } \\
\text { anual } \\
\text { (kWh/año) }\end{array}$ & $\begin{array}{c}\text { Consumo } \\
\text { total } \\
\text { anual }\end{array}$ \\
\hline
\end{tabular}

Tabla 1 Formato para censo de cargas eléctricas. Fuente: (del autor)

\section{Monitoreo con analizador de redes}

Una vez identificados los circuitos y departamentos, se procedió a realizar un monitoreo en campo utilizando equipo de medición de calidad de energía. Para realizar el diagnóstico energético, se utilizó un analizador de redes Fluke 435.

\section{Análisis de la información}

\section{Análisis del diagnóstico energético}

Con los datos obtenidos del diagnóstico energético, se procedió a analizar los consumos de las diferentes áreas y los comportamientos energéticos: voltaje, amperaje, frecuencia, armónicos, balance de cargas, factor de potencia y perfil de demanda. 


\section{Determinación de los KPI - e}

Los indicadores clave de desempeño KPI-e que se implementaron a partir de los datos de monitoreo obtenidos, fueron propuestos en (Schmidt, 2016), pero adaptados a un usuario que se encuentra en tarifa de servicio general y cuyo objeto es ser un parque de recreación con servicios diversos dentro de sus instalaciones.

Se calcularon, según la metodología, indicadores de Tipo I, II y III. Los IV y V se proponen ya habiendo realizado el ejercicio de análisis de los ahorros energéticos y retroalimentarlos hacía posibles impactos energéticos. Los cálculos para los mismos se describen a continuación:

\section{- $\quad$ Indicador Tipo I:}

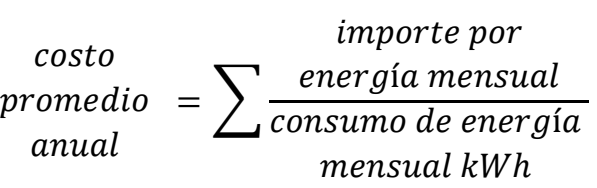

\section{- Indicadores Tipo II:}

$$
\underset{\text { anual }}{\text { consumo }}=\sum \begin{gathered}
\text { consumo de energía } \\
\text { mensual } k W h
\end{gathered}
$$

\section{- Indicador Tipo III:}

$$
\begin{gathered}
\text { emisiones } \\
\text { anuales }
\end{gathered}=\sum\left(\begin{array}{c}
\text { consumo de energía } \\
\text { mensual } k W h
\end{array}\right)(F E)
$$

\section{- Indicador Tipo IV:}

$$
\begin{gathered}
\text { Factor de } \\
\text { potencia }
\end{gathered}=\frac{\begin{array}{c}
\text { consumo de energía } \\
\text { mensual } k W h
\end{array}}{\begin{array}{c}
\text { consumo de } \\
\text { potencia aparente KVA }
\end{array}}
$$

Estos indicadores por su particularidad de integrar información de diferentes niveles y suelen ser utilizados para seguimiento de procesos de producción o diversos usos. En el caso del ejercicio dentro del Parque, fungirán como indicadores para la revisión de los consumos de energía dentro del parque a través de reuniones mensuales con la dirección del parque.

\section{Identificación de áreas de oportunidad}

Con el análisis y determinación de KPI's - e, se identificaron las áreas de oportunidad para priorizar aquellas que requieren una atención a corto plazo, así como proponer estrategias para un consumo más racional y eficiente de la energía.

\section{Propuestas de mejora}

\section{Estrategias para la reducción de consumos}

Las estrategias para la reducción de consumo de energía consisten en las medidas rentables a implementar, las cuales pueden ser: de nula inversión, es decir, ahorros por medidas operativas, y las que necesitan inversión (ahorros por cambio tecnológico) (CONUEE, 2019). Una vez realizado el análisis de datos energéticos e identificados las áreas de oportunidad, se procedió a trabajar en las medidas aplicables a los requerimientos del parque.

\section{Estimación costo - beneficio}

A partir las medidas sugeridas, se hizo una estimación del ahorro esperado, y un comparativo entre el consumo actual y el estimado para determinar el costo - beneficio de la inversión en las mejoras propuestas.

\section{Resultados}

\section{Diagnóstico energético}

\section{Revisión del historial de consumo energético}

En el Gráfico 1 se muestra el consumo energético correspondiente a un año.

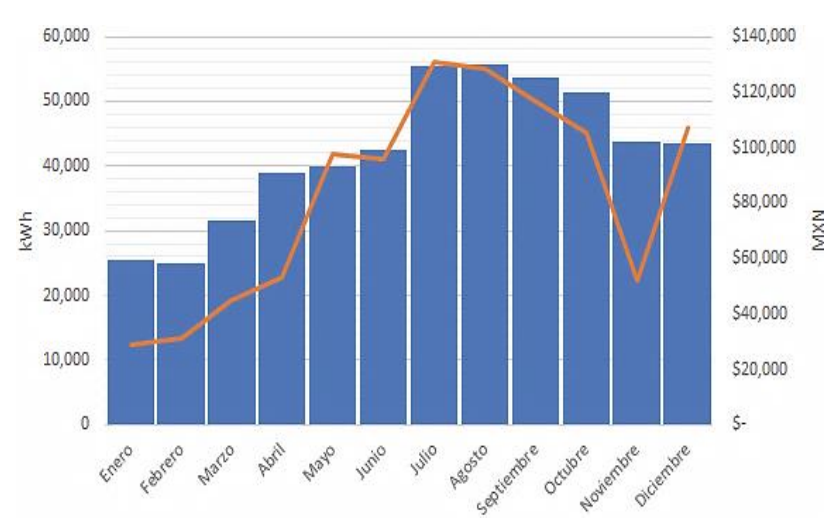

Gráfico 1 Historial de consumo energético

Fuente: Elaboración propia 
Como se aprecia, los meses de mayor consumo son los correspondientes a la época de verano, en los cuales coinciden varios factores que contribuyen a ésto, entre los que se puede mencionar: período vacacional que incrementa el flujo de visitantes, derivando en una mayor actividad y extensión de los horarios de apertura del parque; y las fiestas patrias de la Feria Tijuana, con diversas actividades, en su mayor parte nocturnas, e instalaciones de juegos mecánicos.

\section{Censo de cargas}

El levantamiento del censo de cargas por áreas se muestra en la tabla 2.

\begin{tabular}{|c|c|c|c|}
\hline Concepto & $\begin{array}{c}\text { Consumo } \\
\text { mensual } \\
\text { (kWh/mes) }\end{array}$ & $\begin{array}{c}\text { Consumo } \\
\text { anual } \\
(\mathbf{k W h} / \mathbf{a n ̃ o})\end{array}$ & $\begin{array}{c}\text { Consumo } \\
\text { total } \\
\text { anual } \\
(\mathbf{k W h})\end{array}$ \\
\hline $\begin{array}{l}\text { Subdirección } \\
\text { operativa, } \\
\text { promoción } \\
\text { eventos }\end{array}$ & $1,833.425$ & $22,000.80$ & $524,259.06$ \\
\hline $\begin{array}{l}\text { Dirección, } \\
\text { subdirección } \\
\text { administrativa y } \\
\text { transparencia }\end{array}$ & 2,117 & $25,404.00$ & \\
\hline $\begin{array}{l}\text { Cajas, renta de } \\
\text { kioscos y áreas } \\
\text { verdes }\end{array}$ & 2,892 & $34,704.00$ & \\
\hline $\begin{array}{l}\text { Coordinación de } \\
\text { procedimientos }\end{array}$ & 373 & $4,476.00$ & \\
\hline Contabilidad & 2,663 & $31,956.00$ & \\
\hline Clínica & 717.840 & $8,613.60$ & \\
\hline Granja & $3,291.327$ & $39,495.60$ & \\
\hline Herpetario & 5,093 & $61,116.00$ & \\
\hline Concesionarios & $16,474.255$ & 197,691 & \\
\hline Luminarias & $8,233.5$ & 98,802 & \\
\hline
\end{tabular}

Tabla 2 Censo de cargas por áreas Fuente: Elaboración propia

Las áreas que presentan un mayor consumo son: el herpetario, las luminarias y los concesionarios (ver figura 2). En el caso del herpetario, corresponde al área en la que se encuentran los reptiles. Algunos de éstos se encuentran al aire libre en estanques, como los cocodrilos y las tortugas, pero otros, como las serpientes, iguanas y camaleones, se mantienen en compartimentos tipo incubadora (ver figura 3) que permitan conservar las condiciones climáticas adecuadas para las especies de sangre fría. Cabe mencionar que dichos compartimentos están en funcionamiento las 24 horas del día, los siete días de la semana.

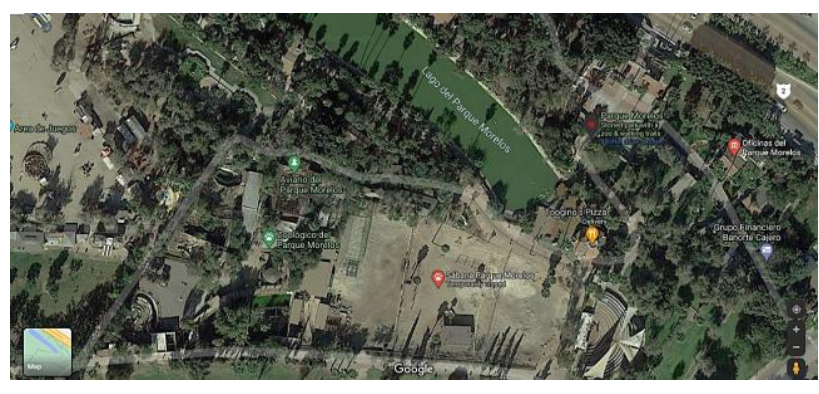

Figura 2 Ubicación de las áreas del censo de cargas: 1. Subdirección operativa, promoción y ventas; 2. Dirección, subdirección administrativa y transparencia; 3 . Cajas, renta de kioscos y áreas verdes; 4. Coordinación de procedimientos; 5. Contabilidad; 6. Clínica; 7. Granja; 8. Herpetario; 9. Área de concesionarios Fuente: Google maps

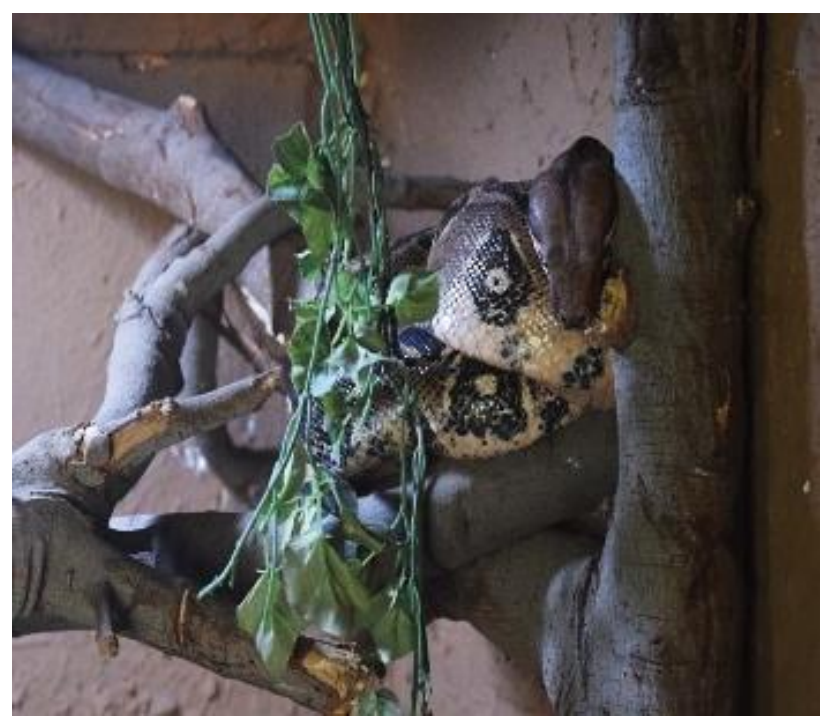

Figura 3 Herpetario del parque Morelos Fuente: (ParqueMorelos.com, S.F.)

Las luminarias representan el segundo mayor consumo identificado en el censo energético. Hay al menos seis tipos diferentes, entre las que predomina el modelo OV - 15 para la iluminación de los espacios abiertos del parque. En su mayoría, son luminarias que se encuentran en condición precaria, por falta de limpieza y mantenimiento.

Respecto a los concesionarios, hay alrededor de 80 puestos, fijos y semifijos, que ofrecen diversas actividades al público: comida, entretenimiento, artísticas, entre otras. El principal problema detectado en este rubro es que no se tiene una política de consumo y sistema de medición de energía. Por ejemplo, fue frecuente observar en los puestos de alimentos equipo de refrigeración con fácil acceso para venta de refrigerios y bebidas; la mayor parte de estos equipos suelen ser obsoletos y no cuentan con sellos de consumo eficiente de energía. 
Otros aspectos observados en el resto de las áreas: uso de contactos múltiples en oficinas, equipos conectados a tomacorriente sin estar en uso, cableado expuesto a la intemperie y empalme de conductores solamente con cinta aislante (ver figura 4).

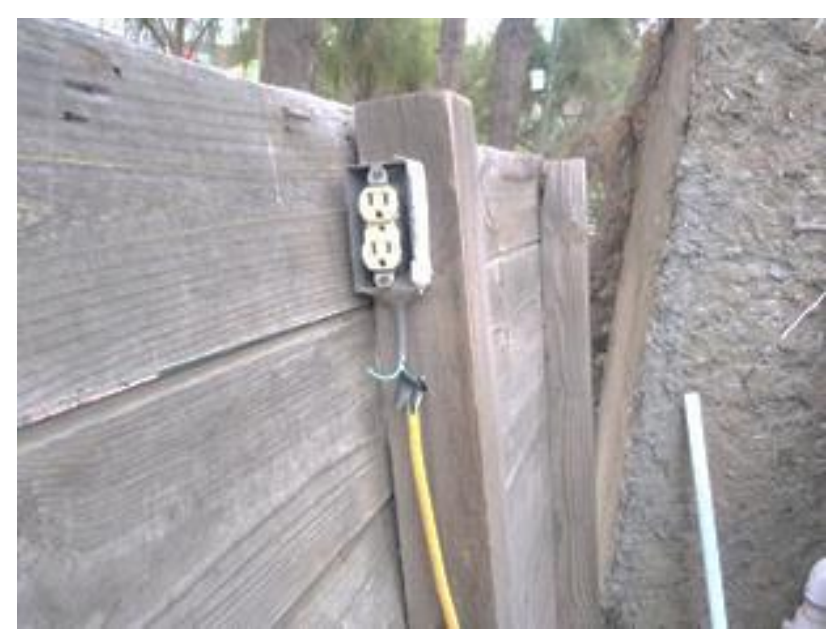

Figura 4 Cableado expuesto y parchado

Fuente: Elaboración propia

\section{Monitoreo con analizador de redes}

La campaña de medición y monitoreo se llevó a cabo durante los días de fin de semana para poder recabar la mayor cantidad de eventos posibles en un intervalo de 10 minutos. Posteriormente los datos del analizador se manipulan con el Software de Fluke® PowerLog® para poder visualizar de manera gráfica los aspectos más relevantes del muestreo. En los gráficos 1 a 3 se observa el comportamiento monitoreado durante un fin de semana típico, que es cuando se tiene mayor actividad en el parque.

En el Gráfico 2 se observa que se presenta un máximo de corriente en L1 de 123 A y un mínimo de 66 A, mientras que en L2 se observa un máximo de 113 A y un mínimo de $58 \mathrm{~A}$, del mismo modo, en L3 un máximo de 138 A y un mínimo de $61 \mathrm{~A}$, demostrando un aumento representativo de las líneas en promedio de $10 \mathrm{~A}$ y una disminución de 4 A.

En el Gráfico 3, se puede observar el comportamiento de voltaje, dándonos como resultado que, al ser fin de semana, hay flujo significativo de personas que ingresan al parque, y esto ocasionó que se requiriera un alto voltaje llegando a los $480 \mathrm{~V}$, el cual está representado en línea roja, y también provocó un incremento de los amperes en el arranque de las bombas y en los motores.
En el análisis del comportamiento de los armónicos, se determinó que no presentan problemas con los armónicos de orden 3, 5 y 7 como se muestra en el Gráfico 4.

La distorsión armónica total (THD) es la que nos dicta las condiciones en las que nuestros sistemas operan por lo que no debe sobrepasar un $2 \%$ de acuerdo con las recomendaciones de IEEE 519 (IEEE, 2014), ya que esto puede provocar calentamiento en las líneas o disturbios a algunos equipos, las instalaciones se encuentran dentro del rango permisible teniendo un $1.54 \%$ como máximo.

En general, es posible visualizar que la demanda máxima del parque en fin de semana llega a 138 Amperes en una de sus Fases (L3). Aparte de encontrarse en desbalance las fases (L1 y L2), la tarifa para fin de semana en GDMTH, es del tipo intermedia para un horario de 00:00 a 24:00 hrs. Por lo que es representativamente más costoso tener una carga máxima en fin de semana a otros dìas entre semana.

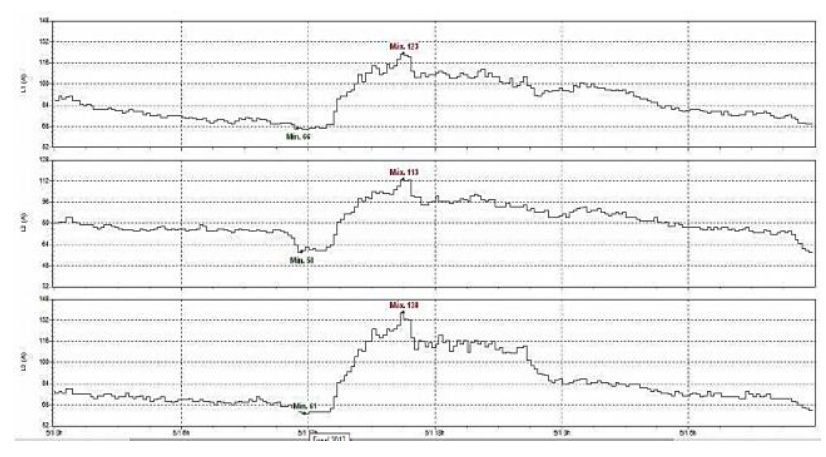

Gráfico 2 Comportamiento de la corriente en fin de semana típico

Fuente: Elaboración propia

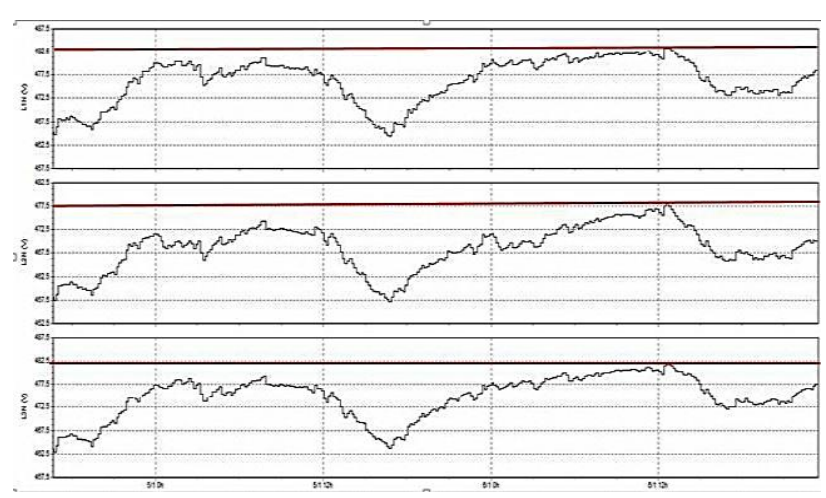

Gráfico 3 Comportamiento del voltaje en fin de semana típico

Fuente: Elaboración propia 


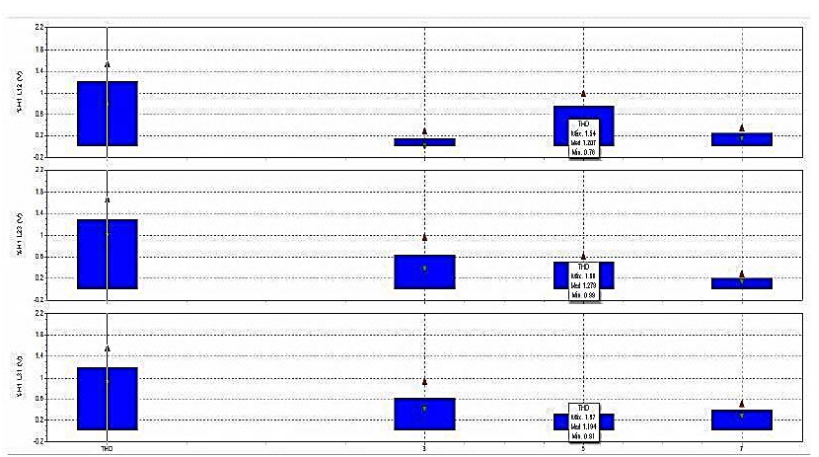

Gráfica 4 Comportamiento de armónicos en fin de semana típico

Fuente: Elaboración propia

\section{Análisis de la información}

\section{Análisis del diagnóstico energético}

\section{Determinación de los KPI - e}

En la tabla 3 se muestran los KPI - e determinados con los datos de consumo energético.

\begin{tabular}{|c|c|c|c|c|}
\hline No. & Nombre KPI - e & Tipo & Nivel & Indicador \\
\hline 1. & $\begin{array}{l}\text { Costo promedio } \\
\text { anual de energía }\end{array}$ & I & Global & $\begin{array}{l}\$ 1.89 \\
\mathrm{MXN}\end{array}$ \\
\hline 2. & $\begin{array}{l}\text { Consumo anual de } \\
\text { energía }\end{array}$ & II & Global & $\begin{array}{l}524,259 \\
\mathrm{kWh}\end{array}$ \\
\hline 3. & Emisiones anuales & II & Global & $\begin{array}{l}264.75 \\
\mathrm{tCO}_{2} \mathrm{e}\end{array}$ \\
\hline 4. & Factor de potencia & III & Global & $73.90 \%$ \\
\hline
\end{tabular}

Tabla 3 Valores de KPI - e determinados

Fuente: Elaboración propia

Obteniendo los KPI-e es importante la interpretación con la finalidad de establecer mejoras y detectar las áreas de oportunidad. En (1.) es posible analizar que el costo promedio anual es de $\$ 1.87$ mientras que el costo de la tarifa intermedia en GDMTH es de $\$ 0.84$, lo cual da la pauta a que es costoso el consumo anual en general.

En (2.) el consumo anual documentado en recibos de CFE es de 506,842 kWh, mientras el dato generado a través de un censo de cargas es de 524,259 kWh. En (3.) las emisiones de GEI equivalentes apuntan al impacto ambiental del consumo de energìa traducido a $\mathrm{CO}_{2}$ equivalente. $\mathrm{Y}$ en (4.) es posible visualizar que el factor de potencia es bajo e inclusive CFE lo penaliza por lo que es un indicador de desempeño que nos indica que se está llevando a cabo un uso ineficiente de la energía.

\section{Identificación de áreas de oportunidad}

Como se pudo observar en la tabla 2, las áreas a priorizar son las de concesionarios y luminarias, para propuestas de medidas operativas y cambio tecnológico por equipos más eficientes en el consumo de energía.

\section{Propuestas de mejora}

\section{Estrategias para la reducción de consumos}

Con los concesionarios, las medidas aplicables son mayormente operativas, ya que los cambios de equipos requieren de una inversión, la cual no todos estarían en condiciones de efectuarla. Se puede realizar una campaña de pláticas para la concientización en el uso y consumo de la energía, así como mostrar un comparativo de mejoras con cambios de hábitos $\mathrm{y}$, para quienes fuera factible, cambio de equipos por otros más eficientes. También se requiere promover una política de consumo de energía por parte de la administración del parque para tener un mayor control en este rubro.

Respecto a las luminarias, y considerando que el sistema de iluminación del parque no se encuentra en las mejores condiciones, conviene realizar un estudio de iluminación con la finalidad de determinar si éstas cumplen con los requerimientos normativos aplicables. El reemplazo tecnológico por modelos más recientes y eficientes en el consumo de energía que pudieran redituar hasta un $29 \%$ de ahorro del consumo actual, representa una de las alternativas más viables.

En el caso del herpetario, debido a que va en juego la conservación de las especies que dependen del acondicionamiento de la temperatura en el hábitat que se encuentran, lo recomendable es realizar un estudio más detallado para una propuesta de reemplazo.

\section{Agradecimiento}

Este proyecto fue autofinanciado por los estudiantes de Ingeniería en Energías Renovables de la Universidad Tecnológica de Tijuana. Agradecemos la colaboración de la I.E.R. Sahian Corey Loreto Escalante, por su contribución en el desarrollo de la parte operativa de este proyecto. 
También se agradece a la dirección de SIMPATT por el acceso a realizar las mediciones, acceso a información y disponibilidad para llevar a cabo el estudio. Y, a la UTT por el acceder a llevar a campo el equipo de medición y realizar las pruebas correspondientes.

\section{Conclusiones}

Fue posible obtener un diagnóstico energético tipo DEN I de las instalaciones del Parque. Se localizaron los usos y usuarios de mayor intensidad energética. A partir de los datos de monitoreo se obtuvo la curva de demanda de energía. Se distinguió los usuarios que preponderantemente deben de seguir políticas energéticas y entrar a algún mecanismo dentro de las posibilidades del parque para retribuir en forma económicamente apropiada por su consumo. Finalmente se proponen proyectos de ahorro de energía las cuales van encauzadas a dos áreas: concesionarios e iluminación, y recomendaciones para el área del herpetario. Dado que regular a los concesionarios requiere de materia administrativa-interna, caen en esquemas de nula inversión a mediano plazo. En cuestión de iluminación, las alternativas de sustitución pueden generar ahorros hasta un 29\% del consumo. En cuestión del herpetario, requiere más estudio la elaboración de una propuesta por tratarse del cuidado de especies en las cuales es crítico el nivel de temperatura.

\section{Referencias}

CONUEE, (2019) "Guía para elaborar un diagnóstico energético en inmuebles"

IEEE, (2014) IEEE Std 519 TM-2014 IEEE Recommended Practice and Requirements for Harmonic Control in Electric Power Systems

ParqueMorelos.com (s.f.), recuperado el 06 de julio de 2020, URL https://www.parquemorelos.com/

Schmidt, C., Li, W., Thiede, S., Kornfeld, B., Kara, S., \& Herrmann, C. (2016). Implementing Key Performance Indicators for Energy Efficiency in Manufacturing. Procedia CIRP, 57, 758-763.

https://doi.org/10.1016/j.procir.2016.11.131
SENER, (2019); Programa de Desarrollo del Sistema Eléctrico Nacional 2019-2033. https://www.gob.mx/sener/documentos/prodese n-2019-2033 